\title{
Hardness and Stress of Amorphous Carbon Films Deposited by Glow Discharge and Ion Beam Assisting Deposition
}

\author{
F. C. Marques and R. G. Lacerda \\ Instituto de Física "Gleb Wataghin", Universidade Estadual de Campinas \\ Caixa Postal 6165, 13083-970, Campinas, São Paulo, Brazil
}

Received 31 January, 2000; received in final form on 17 March, 2000

\begin{abstract}
The hardness and stress of amorphous carbon films prepared by glow discharge and by ion beam assisting deposition are investigated. Relatively hard and almost stress free amorphous carbon films were deposited by the glow discharge technique. On the other hand, by using the ion beam assisting deposition, hard films were also obtained with a stress of the same order of those found in tetrahedral amorphous carbon films. A structural analysis indicates that all films are composed of a $s p^{2}$-rich network. These results contradict the currently accepted concept that both stress and hardness are only related to the concentration of $s p^{3}$ sites. Furthermore, the same results also indicate that the $s p^{2}$ sites may also contribute to the hardness of the films.
\end{abstract}

\section{Introduction}

The carbon atom is probably the most versatile element of nature. Besides its paramount importance in most living beings and organic compounds, a large variety of structures based on the carbon atom have been discovered in nature such as diamond and graphite or artificially developed structures such as those of buckballs, nanotubes, and diamond-like carbon thin films $[1,2]$. The ability of carbon to hybridize in $s p, s p^{2}$ and $s p^{3}$ configurations is not a common characteristic of most elements. This peculiarity limits the establishment of universal models to explain the large variety of structures found in those materials. For instance, one of the main difficulties faced by many researchers is to understand the origin of the intrinsic stress and hardness of amorphous carbon films.

Diamond is one of the hardest materials found in nature. Its high hardness is attributed to strong C$\mathrm{C} s p^{3}$ bonds in a tetrahedrally coordinated network. On the other hand, the softness of graphite is associated with a structure of planes formed by closed-packed rings of $s p^{2}$-hybridized carbon atoms. In the preparation of amorphous carbon films one usually finds a mixture of $s p^{2}$ and $s p^{3}$ bonds. The hardness of amorphous carbon films has been currently attributed to the concentration of $s p^{3}$ sites present in the films. In addition, the stress of those films has also been associated with the concentration of $s p^{3}$ sites created during the film growth $[3,4]$. Therefore, direct relations be- tween hardness and stress with the $s p^{3}$ concentration have been established and frequently used in literature $[5,6]$. In this paper we show that the explanation for the structural origin of the hardness and the stress of amorphous carbon films in terms of the concentration of $s p^{3}$ bonds is not universal. We observed, using two different kinds of deposition systems, that it is possible to prepare hard amorphous carbon films with high concentrations of $s p^{2}$ bonds. In addition, we demonstrate that it is also possible to prepare highly stressed films with small concentrations of $s p^{3}$ bonds.

\section{Experimental}

The films were prepared using two different techniques, i.e., glow discharge (GD) and Ion Beam Assisting Deposition (IBAD). A first series of films were deposited on the cathode electrode of a GD system through a plasma decomposition of methane gas. All films were prepared at room temperature. The bias voltage was varied within the range $-100 \mathrm{~V}$ to $-1200 \mathrm{~V}$ and the $\mathrm{CH}_{4}$ gas pressure was kept constant at 1.0 Pa. A second series of films was prepared by IBAD using two Kauffman ion sources. Detailed descriptions of the deposition system are found elsewhere [7]. Argon gas was introduced into both Kauffman ion sources for the sputtering of a graphite target and for the simultaneous assisting of the growing film. The graphite target was sputtered by a $1500 \mathrm{~V}$ argon ion beam with a total current of $90 \mathrm{~mA}$. 
A set of films was prepared at $150 \mathrm{C}^{\circ}$ in the range of 0 to $800 \mathrm{eV}$ argon ion beam assisting energy. The base pressure of the chamber was about $10^{-5} \mathrm{~Pa}$, and during deposition the pressure was kept constant at $6 \times 10^{-2}$ Pa.

The thickness of the films, measured by a Dektak profilometer, was about $1 \mu \mathrm{m}$ for the films prepared by GD and about $0.1 \mu \mathrm{m}$ for those prepared by IBAD. The hardness was obtained from a nanoindenter using a set of different loads in the $0.5 \mathrm{mN}$ to $90 \mathrm{mN}$ range. The Raman spectroscopy was obtained at room temperature with a T64000 Jobin Ybon spectrometer in the backscattering configuration. Stress measurements were taken from films deposited on $4 \times 25 \times 0.4 \mathrm{~mm}^{3}$ c-Si bars, using the bending beam method to determine the radius of curvature of the film/substrate composite [8]. The stress was then calculated using the modified Stoney's equation $[9,10]$ :

$$
\sigma=\left[E_{s} /\left(1-\nu_{s}\right)\right] t_{s}^{2} / 6 t_{f}\left(1 / R-1 / R_{o}\right)
$$

where $E, \nu$, and $t$ are the Young;s modulus, Poisson's ratio, and thickness, respectively. The subscripts $s$ and $f$ refer to the substrate and the film, respectively. $1 / R_{o}$ and $1 / R$ are the curvatures before and after the film deposition.

\section{Results}

Fig. 1 displays the deposition rate of the two series of films prepared by glow discharge and IBAD. Observe that the deposition rate of the films prepared by GD is much higher than that of the films prepared by IBAD. The increase of the deposition rate with the bias voltage of the films prepared by GD is attributed to an increase of the decomposition rate of the methane gas. On the other hand, in the IBAD technique the deposition rate is determined by the target-sputtering yield and by the assisting ion energy. Even though the flux of sputtered species from the graphite target was kept constant, the assisting ions play an important role for determining the deposition rate since they sputter the growing film. Hence, as the assisting ion energy increases the deposition rate reduces.

Hard amorphous carbon films are usually obtained at very low deposition rates, typically at about 0.01 $\mathrm{nm} / \mathrm{s}[11,12]$. However, Fig. 2 shows that in the case of glow discharge, it is possible to achieve high deposition rates and also relatively high hardness. This result is very import for industrial application and it may depend on the deposition system as well as on its geometry. On the other hand, even though the films prepared by IBAD have hardness of about $30 \mathrm{GPa}$, their deposi- tion rates are very low, which hinders their use in many industrial applications.

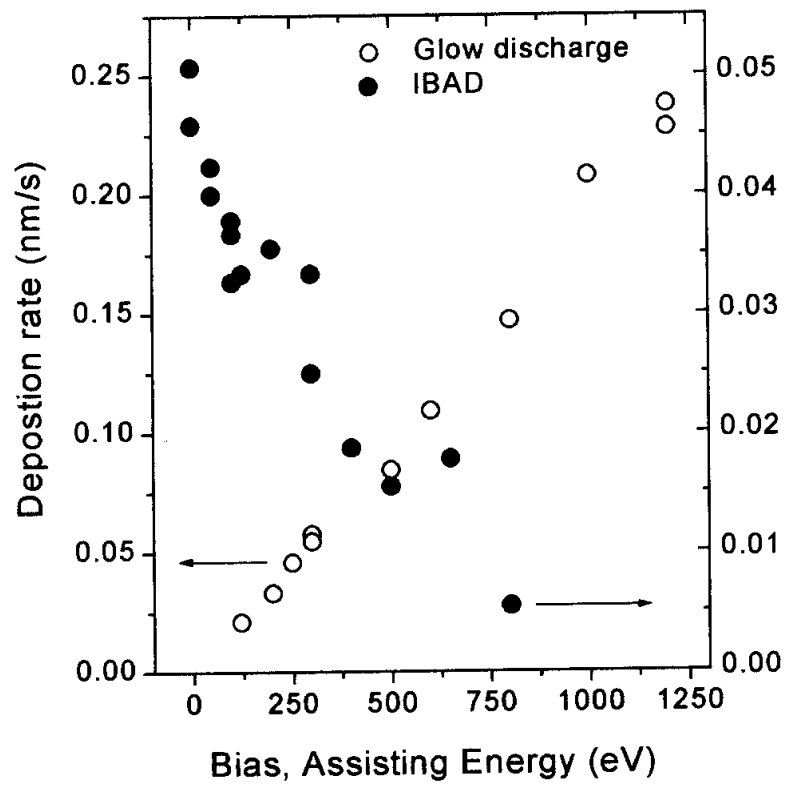

Figure 1. Deposition rates of amorphous carbon films as a function of the bias voltage (for films prepared glow discharge) and the assisting energy (for films prepared by ion beam assisting deposition). Observe the difference in the scale of both series.

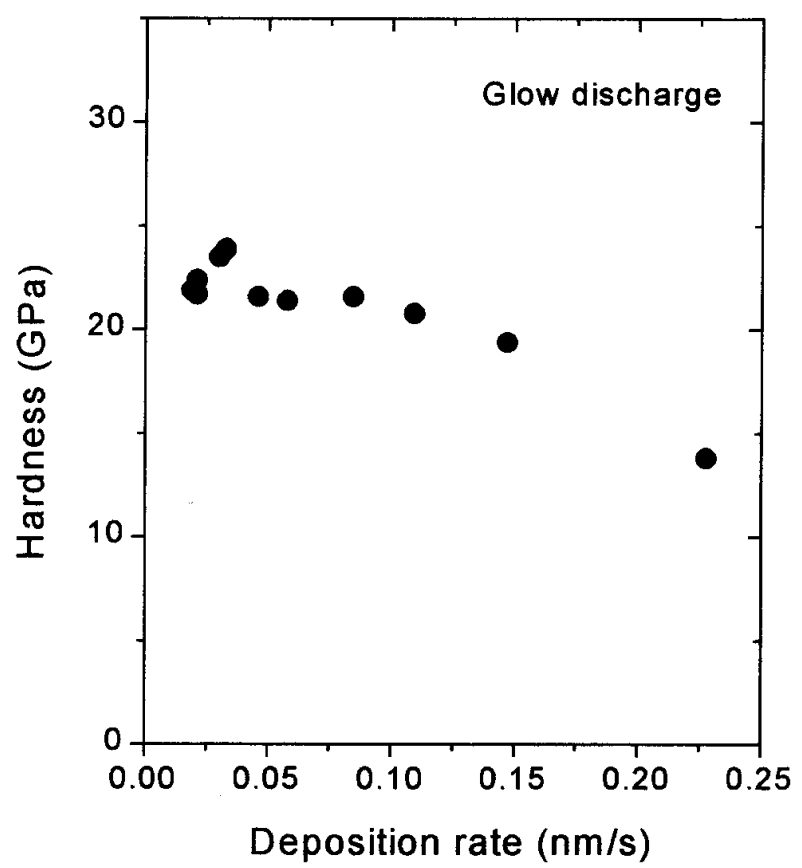

Figure 2. Hardness versus deposition rate of amorphous carbon films prepared by glow discharge, deposited at different bias voltages.

Fig. 3 shows the compressive intrinsic stress as a function of the bias (GD) and the assisting energy (IBAD). The stress curve of the films prepared by GD presents a maximum close to $120 \mathrm{eV}$, and the stress are 
of the same order of those typically obtained in hard amorphous carbon films (2-3 GPa). For higher bias voltage the stress decreases substantially reaching values as low as $0.5 \mathrm{GPa}$. The stress of the films deposited by the IBAD increases sharply for argon assisting energy up to $100 \mathrm{eV}$, and it varies slightly for energy in the $100 \mathrm{eV}$ to $650 \mathrm{eV}$ range. In addition, the absolute value of the stress is of the same order of those reported for films with high concentrations of $s p^{3}$ bonds (ta-C) $[5,13]$. Even though the stresses of both series of films are very different, the hardness of those films is about the same, Fig. 4. This figure shows that the hardness changes just about a factor of two while the stress differs by more than one order of magnitude.

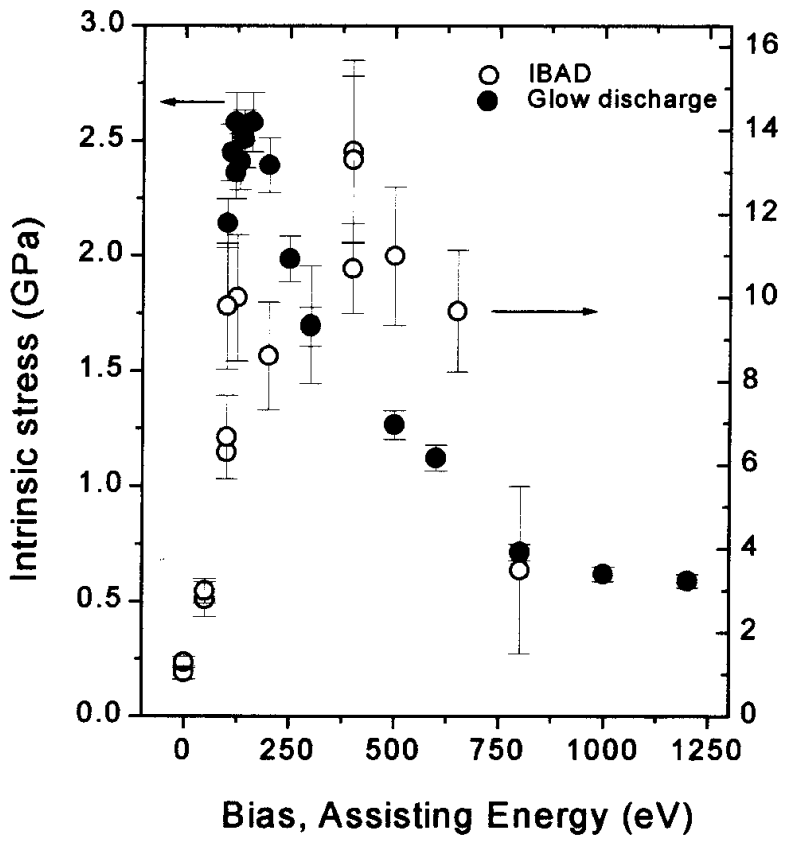

Figure 3. Stress of amorphous carbon films as a function of the bias voltage (for films prepared by glow discharge) and the assisting energy (for films prepared by ion beam assisting deposition).

All films reported in this work are characterized by having a high concentration of $s p^{2}$ hybridization as indicated by the structural analysis of the Raman spectroscopy, Fig. 5. This figure shows typical Raman spectra of two films deposited by GD and IBAD. They clearly show the disorder band (D) at $\sim 1340 \mathrm{~cm}^{-1}$, and the graphite band $(\mathrm{G})$ at $1530 \mathrm{~cm}^{-1}$. The ratio between the areas of these two bands, $I_{d} / I_{g}$, indicates the presence of $s p^{2}$ sites in the film structure [14]. Fig. 6 shows the dependence of the $I_{d} / I_{g}$ on both the bias voltage (GD) and the assisting energy (IBAD). For films prepared by GD, one observes an increase of the $I_{d} / I_{g}$ ratio as the bias increases, which indicates an increase of the concentration of the $s p^{2}$ sites. Although no trend had been observed for films deposited by IBAD as a function of the assisting energy, they also have a high value of $I_{d} / I_{g}$ ratio of about 2 which also indicates a graphiticlike structure. The results of both series contrasts with the low values of the $I_{d} / I_{g}$ ratio $(\sim 0.1)$ found in high tetrahedral amorphous carbon films [15].

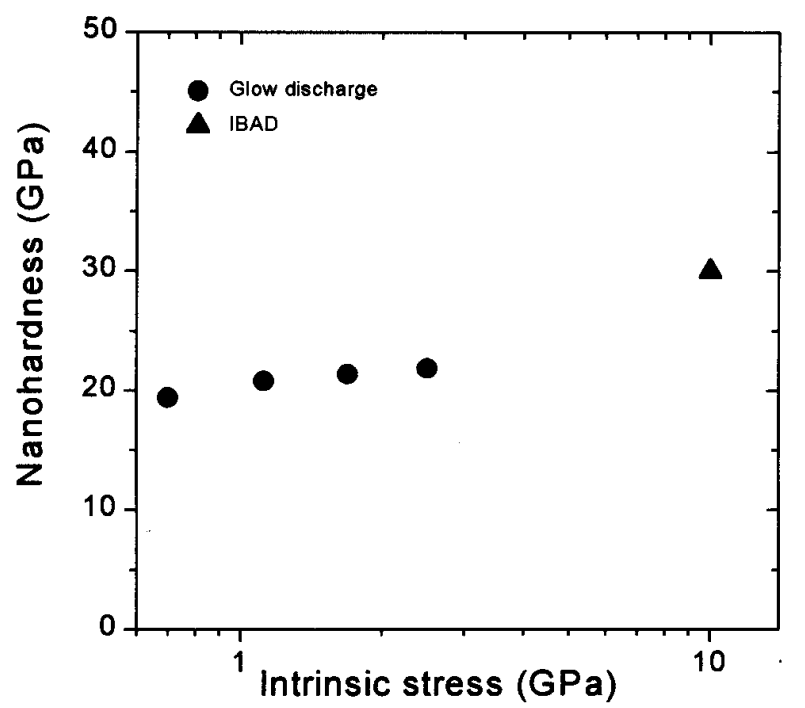

Figure 4. Hardness versus stress of several amorphous carbon films prepared by glow discharge and ion beam assisting deposition.

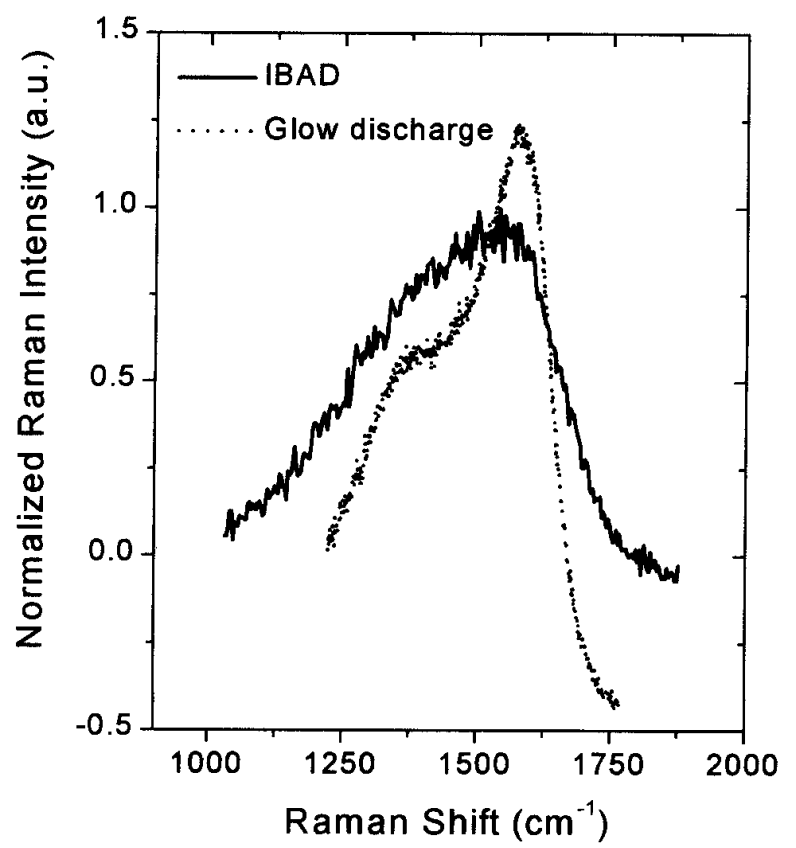

Figure 5. Typical Raman spectra of amorphous carbon films prepared by glow discharge and ion beam assisting deposition.

The density of the films is in the $1.5-2.25 \mathrm{~g} / \mathrm{cm}^{2}$ range, Fig. 7. Within experimental error, there is no clear trend for films prepared by GD. However, there is a broad range of assisting energy giving higher density 
in the $100-600 \mathrm{eV}$ range. These densities are smaller than those reported for tetrahedrally coordinated amorphous carbon films (ta-C), with density of about 3 $\mathrm{g} / \mathrm{cm}^{3}$ [16]. It is worth noting that some of the films prepared by IBAD have a stress of the same order of those found in ta-C films (typically of the order of 10 $\mathrm{GPa}$ ), see Fig. 8. However, the density of these films is much higher than the density of the films developed in this work. This result is very different from what has been reported in the literature, i.e., films with very high stress have high density.

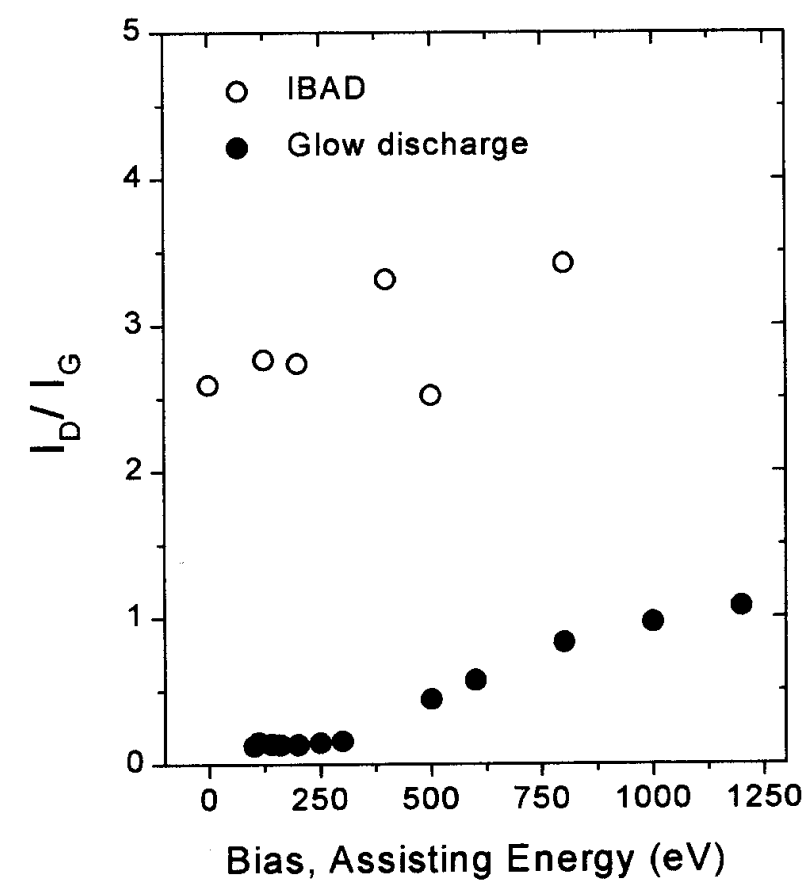

Figure 6. Raman, $I_{d} / I_{q}$, ratio of amorphous carbon films prepared by glow discharge and ion beam assisting deposition as a function of the bias voltage and the assisting energy, respectively.

\section{Discussions}

For amorphous carbon films, there are some intriguing results obtained in our work that challenge the validity of some theoretical models reported in the literature. 1) the films clearly have a structure with high concentrations of $s p^{2}$ hybridization, yet they are relatively hard; 2) It was possible to prepare such kind of films with very low stress as well as films with very high stress; 3 ) films with high hardness and low stress could also be prepared with relatively high deposition rates. Let us address those features.

First, it is important to stress that our films are characterized by having a very high concentration of $s p^{2}$ sites. In other words, they are not tetrahedrally coordinated amorphous carbon films. The Raman results are important to determine this characteristic. As it is well know, the Raman scattering has been used to identify the presence of $s p^{2}$ and $s p^{3}$ hybridization. Even though this technique is not the best way for measuring the $s p^{2} / s p^{3}$ ratio, it has been used as a qualitative measure of the degree of graphitization of the films. As we observed in Figs. 5 and 6 , all films are characterized by a relatively high $I_{d} / I_{g}$ ratio, indicating that the concentration of $s p^{2}$ sites is very high. These results are also supported by the small band gap of those films, which is typically smaller than $1.0 \mathrm{eV}$, and also by the relatively small density of less than $2.3 \mathrm{~g} / \mathrm{cm}^{3}[17,18]$.

It is well accepted that the hardness of highly tetrahedrally coordinated carbon films is due to the presence of $s p^{3}$ bonds. However, in this work we developed films with high concentrations of $s p^{2}$ sites but with relatively high hardness (15-30 GPa). This result contradicts the currently accepted concept that the hardness of amorphous carbon films depends only on the concentration of $s p^{3}$ sites, even for films with high concentrations of $s p^{2}$ sites. Therefore, we propose that the $s p^{2}$ sites, if they are appropriately distributed, may also contribute to the hardness of materials such as crosslinked $[19,20]$ or curved graphitic sheet structures [21]. It is important to stress this contribution, since graphite has a very small hardness. However, its hardness comes from an organized network, which allows an easy dislocation of parallel planar layers and hence reducing the hardness of the material. An appropriated distribution of the carbon rings can be developed in a way to eliminate this problem and provide a rigid network. One should bear in mind that the $s p^{2}$ bond is in fact stronger than the $s p^{3}$ bond, so it is not strange to assume that somehow the $s p^{2}$ sites could also contribute to the hardness of the films.

Second, it has been claimed that the hardness of amorphous carbon films is related to the stress since the same process of sub-implantation has been used to explain both stress and hardness through the formation of $s p^{3}$ sites [3-5]. We intend to show here that this is not always valid. Fig. 7 shows a plot of hardness versus stress for a selected set of films prepared by both techniques. It is amazing to note that films prepared with a stress as low as $0.5 \mathrm{GPa}$ have hardness of about the same order of that of films which have stress of more than one order of magnitude higher. It is important to remember that films with a stress of about the same order of those obtained for ta- $\mathrm{C}$ were developed by IBAD. In other words, we show that it is also possible to prepare highly stressed and hard films with high concentrations of $s p^{2}$ bonds. In the present series of films we propose that the intense argon bombardment 


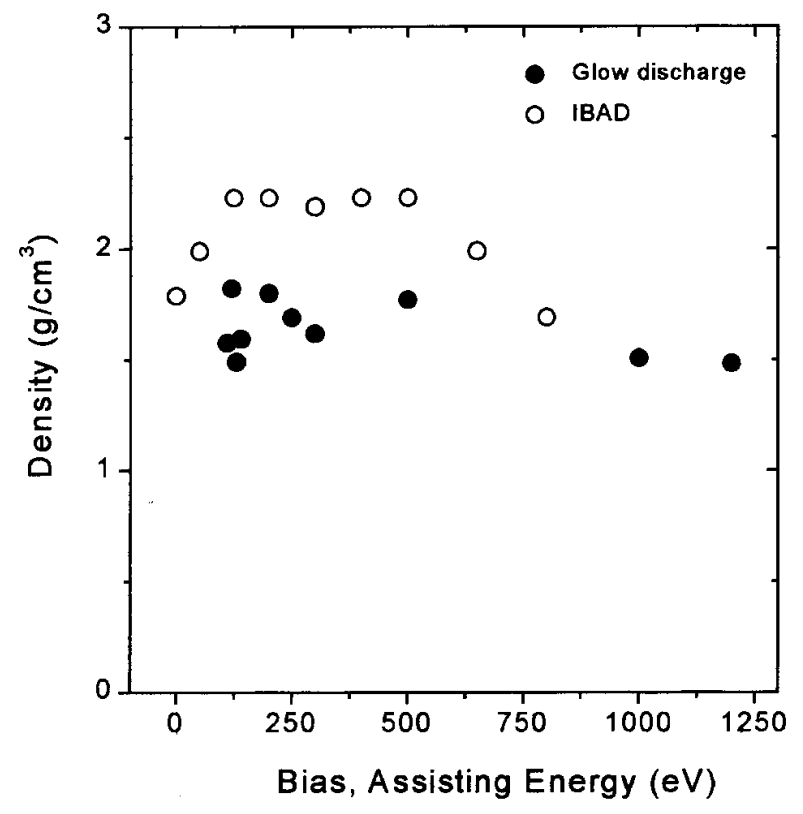

Figure 7. Density of amorphous carbon films as a function of the bias voltage (for films prepared by glow discharge) and the assisting energy (for films prepared by ion beam assisting deposition).

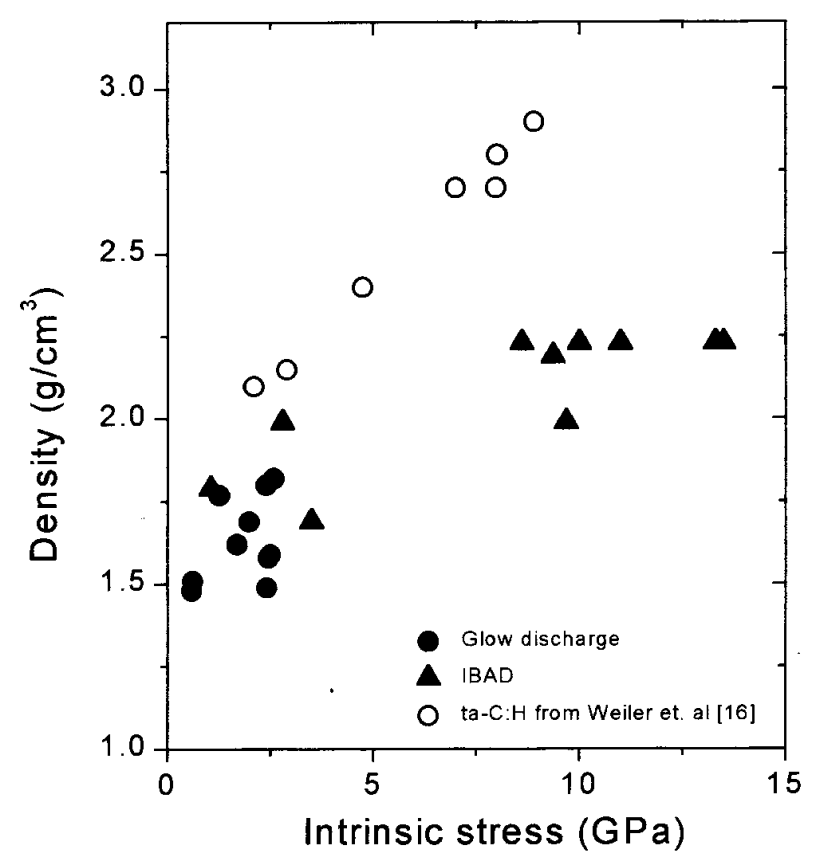

Figure 8. Density versus intrinsic stress of amorphous carbon films prepared by glow discharge and ion beam assisting deposition. A series of tetrahedrally coordinated amorphous carbon (ta-C) films is also included for comparison [16].

and the subsequent carbon implantation compact the films, generating high compressive stress in a dense $s p^{2}$ structure [18]. This result brings back the debate on the explanation for the origin of the stress in amorphous carbon films. They show that there are no unique relations among stress, hardness and $s p^{3}$ concentration. In addition, the presence of hydrogen, as in the case of the films deposited by glow discharge, must also be taken into account, since they are important for determining the structure of the films. Among other contributions, hydrogen could relieve the stress and hinder the formation of large clusters. Hence, with the influence of hydrogen, it is reasonable to assume that it is possible to obtain films with the same $s p^{2} / s p^{3}$ ratio but with very different properties, such as hardness and stress.

Finally, in Fig. 2 we observed that it was possible to prepare films at high deposition rates with reasonably high hardness and low stress. These results are of extreme importance for industrial application. Recently, we have also developed good quality films by the glow discharge technique with an even higher deposition rate $(\sim 0.7 \mathrm{~nm} / \mathrm{s})$ using higher methane gas pressure and bias voltage [22]. It is very likely that these data can still be improved by using more appropriated conditions. For instance, some reduction of stress could be achieved by the introducing of nitrogen and boron into the carbon network $[23,24]$. This procedure could be used together with the condition adopted here to improve the film properties, especially for films prepared at high bias.

In summary, the study of amorphous carbon films has emphasized mainly the achievement of highly tetrahedrally coordinated carbon films. Usually, these films have a hardness near that of diamond but they are very highly stressed and deposited at very low deposition rate. Hence, these films are not good candidates for most applications, since they can not be prepared at an appropriate thickness because of their low deposition rate and high stress. In this work we show that it is possible to prepare films with a relatively high hardness, low stress and high deposition rate. Even though their hardness is not as high as those reported for ta- $\mathrm{C}$ films, the films prepared by GD are hard enough for many industrial applications.

\section{Conclusion}

In this work we demonstrated that it is possible to prepare amorphous carbon films by the glow discharge technique with a low stress, a relatively high hardness and a high deposition rate. These films are good candidates for industrial applications. They are $s p^{2}$-rich with a structure that somehow provides high hardness, such as crosslinked or curved graphitic sheets. On the other hand, we could also prepare highly stressed films with similar characteristics using the ion beam assisting deposition (IBAD) technique. These results demonstrate that there are no unique relations among stress, 
hardness and $s p^{3}$ hybridization. Such type of relations reported in the literature may apply to some specific cases but they can not be generalized.

\section{Acknowledgment}

The authors are indebt to F. Alvarez and P. Hammer for fruitful discussions, to F. L. Freire Jr. for RBS measurements and to C. M. Lepienski for nanoindentation measurements.

\section{References}

[1] J. Robertson, Prog. Solid St. Chem. 21, 199 (1991).

[2] D. R. McKenzie, Rep. Prog. Phys. 59, 1611 (1996).

[3] C. A. Davis, Thin Solid Films 226, 30 (1993).

[4] J. Robertson, Diamond and Relat. Mat. 2, 984 (1993).

[5] D. R. MacKenzie, D. Muller and B. A. Pailthope, Phys. Rev. Lett. 67, 773 (1991).

[6] J. Robertson, Diamond and Relat. Mat. 3, 361 (1994).

[7] P. Hammer, N. M. Victoria and F. Alvarez, J. Vac. Sci. and Technol. A 16, 2491 (1998).

[8] M. M.de Lima Jr., R. G. Lacerda, J. Vilcarromero and F. C. Marques J. of Appl. Phys. 86, 4636 (1999).

[9] Mary F. Doerner, Willian D. Nix, CRC Critical Review in Solid State and Materials Sciences 14, (3), 228 (1988).

[10] W. A. Brantley, J. Appl. Phys. 44, 534 (1973).

[11] X. Jiang, K. Reichelt and B. Stritzker, J. Appl. Phys. 66, 5805 (1989).

[12] Z. W. Zou, K. Reichelt, K. Schmidt and B. Dischler, J. Appl. Phys. 65, 3915 (1989).
[13] P. J. Fallon, V. S. Veerasamy, C. A. Davis, J. Robertson, G. A. J. Amaratunga, W. I. Milne and J. Koskinen, Phys. Rev. B 48, 4777 (1993).

[14] J. Schwan, S. Ulrich, V. Batori, H. Ehrhardt and S. R. P. Silva, J. Appl. Phys. 80, 440 (1996).

[15] B. K. Tay, X. Shi, H. S. Tan, H. S. Yang and Z. Sun, Surf. Coat. Technol. 105, 155 (1998).

[16] M. Weiler, S. Sattel, T. Giessen, K. Jung, H. Ehrhardt, V. S. Veerasamy and J. Robertson, Phys. Rev. B 53, 1594 (1996).

[17] R. G. Lacerda, F. C. Marques and F. L. Freire Jr., Diamond Relat. Materials 8, 495 (1999).

[18] R. G. Lacerda, P. Hammer, F. Alvarez, F. C. Marques and F. L. Freire Jr., Diamond Relat. Materials (to be published).

[19] R. G. Lacerda and F. C. Marques, Appl. Phys. Lett. 73, 619 (1998).

[20] F. C. Marques, R. G. Lacerda, G. Y. Odo, C. M. Lepienski, Thin Solid Films 332, 113 (1998).

[21] I. Alexandru, H. -J. Scheibe, C. J. Kiely, A. J. Papworth, G. A. J. Amaratunga and B. Schultrich, Phys. Rev. B 60, 10903 (1999).

[22] F. C. Marques, R. G. Lacerda, M. M. de Lima Jr. and J. Vilcarromero, Thin Solid Films 343-344, 222 (1999).

[23] D. F. Franceschini, C. A. Achete and F. L. Freire Jr., Appl. Phys. Lett. 60, 3229 (1992).

[24] M. Chhowalla, Y. Yin, G. A. J. Amaratunga, D. R. McKenzie and T. Frauenheim, Appl. Phys. Lett. 60, 3229 (1992). 